\title{
ARTE CONTEMPORÂNEA E CULTURA VISUAL: DIÁLOGOS E POSSIBILIDADES NO ENSINO SUPERIOR
}

\author{
Arte contemporáneo y cultura visual: diálogos y posibilidades en la enseñanza superior \\ Contemporary art and visual culture: dialogues and possibilities in the higher education
}

Lutiere Dalla Valle ${ }^{1}$

\begin{abstract}
Resumo
Este texto explora relações entre arte e cultura visual a partir de projetos de ensino, pesquisa e extensão desenvolvidos junto ao Laboratório Artes Visuais e I/Mediações - vinculado ao Departamento de Artes Visuais da Universidade Federal de Santa Maria. Tem por objetivo compartilhar experiências, refletir sobre trajetórias desenvolvidas e contribuir com algumas possibilidades para a formação acadêmica no campo das artes visuais. Pelo viés da perspectiva da cultura visual, esboça articulações inter e transdisciplinares, a partir de projetos em andamento, aqui pensados como potência. Como fundamentação teórico-metodológica, teóricos como Fernando Hernández, Nicolas Mirzoeff, Thomas W. J. Mitchell, Belidson Dias, entre outros, subsidiam projetos e experiências. Em forma de relato, o texto pretende não apenas situar estas trajetórias, mas discutir - à luz do referencial - alguns aspectos pontuais acerca da formação, tecendo ideias entorno às proposições realizadas.
\end{abstract}

Palavras-Chave: Arte Contemporânea; Cultura Visual; Ensino Superior

\section{Resumen}

Este texto explora relaciones entre arte y cultura visual a partir de proyectos de investigación y extensión desarrollados junto al Laboratorio Artes Visuales e In/Mediaciones - vinculado al Departamento de Artes Visuales de la Universidad Federal de Santa María. Tiene por objetivo compartir experiencias, reflexionar sobre trayectorias desarrolladas y contribuir con algunas posibilidades para la formación académica en el campo de las artes visuales. Desde la perspectiva de la cultura visual, esboza articulaciones inter y transdisciplinarias a partir de proyectos en marcha, aquí pensados como potencia. Como fundamento teórico-metodológico utilizamos Fernando Hernández, Nicolas Mirzoeff, Thomas W. J. Mitchell, Belidson Dias, entre otros autores que subsidian los proyectos y experiencias. En forma de relato, el texto pretende no sólo situar estas trayectorias, sino discutir algunos aspectos puntuales sobre la formación, tejiendo ideas en torno a las proposiciones realizadas.

Palabras Clave: Arte Contemporáneo; Cultura Visual; Enseñanza Universitaria

\begin{abstract}
This issues explores relationships between art and visual culture from teaching, research and extension projects developed by the Laboratory of Visual Arts and I / Mediations - Visual Arts Department of the Federal University of Santa Maria. It aims to share experiences, reflect on developed trajectories and contribute with some possibilities for academic training in the field of visual arts. From the perspective of visual culture, it outlines inter and transdisciplinary articulations, from projects in progress, here thought as power. As a theoretical-methodological perspectives from Fernando Hernández, Nicolas Mirzoeff, Thomas W. J. Mitchell, Belidson Dias, and others, subsidize projects and experiences. In the form of an account, the text intends not only to situate these trajectories, but to discuss some specific aspects about the formation, weaving ideas around the realized propositions.
\end{abstract}

Keywords: Contemporary Art; Visual Culture; Higher Education

1 Docente do Programa de Pós-Graduação em Artes Visuais - PPGART/UFSM, Departamento de Artes Visuais da Universidade Federal de Santa Maria. Santa Maria-RS- Brasil,- e-mail: lutiere@dallavalle.net.br 


\section{Introdução}

Como os artistas se dedicam a dar forma à sua experiência vivida, os objetos artísticos são, em certo sentido, experiências vividas transformadas em configurações transcendidas. (VAN MANEN, 2003, p. 92).

Em cada contexto cultural, imagens incitam nosso pensar: nos ajudam a sistematizar, organizar, apreender e difundir relações com/a partir da vida cotidiana atravessada pelas vias da interpretação e da significação que cada contexto nos oferece. Os contornos sociais que movimentam nossas formas de agir e estar no mundo, em muitos casos mediados pelas representações visuais que fazemos, configuram, portanto, nosso olhar, nossa humanidade. Neste sentido, inventamos a arte para, talvez, dimensionar aquilo que nos escapa à linguagem literária (escrita/falada) para abraçar outras formas de compreensão - o que nos abre um leque de infinitas possibilidades. Arriscamos apreender ao estabelecer relações: uma coisa se conecta à outra, tecendo redes de significado (que dentro de um determinado contexto cultural ganha forma e sentido). Assim, "produzir arte, portanto, antes de ser uma capacidade de expressão, configura um ato de consciência" (RESENDE, 2007, P. 59).

Nossas primeiras aprendizagens como humanos acontece dentro da cultura à qual nos vemos implicados: aprendemos a ser homens ou mulheres, absorvemos, da lógica cultural, as suas hierarquias, regras e valores a partir da observação, e principalmente a partir da experimentação, incluindo-se a linguagem comumente legitimada pelo grupo social. Também passamos por diversas etapas e papéis: aprendemos a nos relacionar inicialmente como bebês, e posteriormente como crianças, adolescentes, jovens, adultos, idosos, e, em cada etapa, desenvolvemos formas de ver, relacionar, articular, falar, deslocar, sonhar, planejar, enfim, de compreender o mundo e a nós mesmos.

Neste ínterim, ao argumentar esta escrita em defesa da cultura visual, busco respaldar-me em autores como Nicholas Mirzoeff (2003), ao afirmar que ao nos posicionarmos a partir deste enfoque, estamos levando em consideração o "papel determinante que desempenha a cultura visual na cultura mais ampla a que pertence o sujeito" (p. 21). Sobretudo, em nossa contemporaneidade em que as artes visuais advêm das múltiplas possibilidades e diversidades étnicas e culturais, principalmente das experiências cotidianas ancoradas por imaginários coletivos, configurados a partir das múltiplas mídias.

Tomar a perspectiva educativa da cultura visual como ancoradouro nos favorece interrogar as elaborações discursivas que envolvem a construção do olhar, e também os estereótipos que contribuem para definir condutas, delinear posições hierárquicas e legitimar papéis sociais. Sobretudo, o olhar produzido pela cultura dominante (heteronormativa, branca e eurocêntrica), que tem privilegiado ao longo dos séculos alguns elementos culturais peculiares de forma linear e impositiva como verdade, desconsiderando, em muitos aspectos, as contingências que envolvem a complexidade humana e sua diversidade.

Neste sentido, é possível que as narrativas visuais privilegiem certas posições críticas e inventivas, pois as interpretações que fazemos dos artefatos visuais (imagens da publicidade, obras de arte, televisivas, cinematográficas, etc.) encontram-se afetadas pelos limites impostos pelo enquadramento, pela delimitação material de alguém sobre o que tornar visível e o que deixar fora do campo visual. Isto é, ao pensar com/a partir das imagens, temos em conta que cada sujeito aporta sua própria experiência, história e identidade diante da compreensão de uma imagem. Assim como Mitchell (2009), também acredito que nossas concepções, formas de ver, pensar e articular o mundo, perpassam pelas relações que estabelecemos com as imagens, sobretudo, com aquelas que nos afetam - em maior ou menor grau.

Portanto, a narrativa que segue, parte deste lugar provisório e relacional da cultura visual: em vias de constituição, abordagem que se nutre das experiências vivenciadas cotidianamente pelos sujeitos diante das imagens, reflexos da nossa contemporaneidade imersa nos dispositivos do olhar. Arte contemporânea e cultura visual, enquanto linha de investigação - potência mobilizadora - remota à experiência, à possibilidade da invenção e da autonomia do sujeito diante dos artefatos visuais produzidos pelas culturas, e sobre às infinitas possibilidades de articular a produção artística contemporânea e seus modos de concepção, recepção e difusão. Neste contexto, as ações articuladas dentro do Laboratório Artes Visuais e I/Mediações (LAVI/M) constituem nosso ponto de partida.

\section{Sobre as I/Mediações}

O termo I/mediações empregado neste texto diz respeito às discussões que viemos desenvolvendo no grupo de pesquisa que coordeno (Artes Visuais e suas I/Mediações/CNPq) há alguns anos, ao explorar conexões entre arte, cultura visual, espaços expositivos, projetos educativos, projetos colaborativos e de interação. Inserido na Universidade Federal de Santa Maria - Departamento de Artes Visuais (local em que atuo há alguns anos como docente na formação de artistas, professores e professoras, pesquisadores e pesquisadoras em nível de graduação e pós-graduação). Neste ínterim, a proposição léxica explora possibilidades de compreensão das produções visuais contemporâneas (produzidas a partir das mais variadas fontes) em relação às múltiplas alternativas de pesquisa em arte e visualidade, 
bem como outros desdobramentos vinculados à extensão universitária.

I/mediações refere-se tanto ao que está próximo, quanto ao que está às margens, que está nas imediações, nas proximidades. É dialógico. As delimitações são tênues ou inexistentes. I/mediação diz respeito a tudo aquilo que pode promover profícuas relações a partir dos distintos saberes que têm como fio condutor os artefatos culturais. Mediação, sob este aspecto, implica também estar no meio, apresentar-se como interlocutor, como dispositivo/disparador entorno a determinados artefatos visuais: obras de arte, filmes, imagens publicitárias, etc. I/mediação empreende, portanto, mobilidade, deslocamento entre objeto, visualizador e interpretação. I/mediação ao estabelecer conexões com variados campos - como os estudos da cultura visual que se nutrem de variadas áreas das ciências sociais para compreender as relações diárias com as visualidades - almeja a pluralidade inventiva, o caráter provisional e as qualidades da negociação coletiva em diálogo com a dimensão subjetiva que aporta cada sujeito. A partir do argumento de Belidson Dias (2011)

Nessa direção, a educação da cultura visual é aberta a novas e diversas formas de conhecimentos, promove o entendimento de meios de opressão dissimulada, rejeita a cultura do Positivismo, aceita a ideia de que os fatos e os valores são indivisíveis, e, sobretudo, admite que o conhecimento é socialmente construído e relacionado intrinsecamente ao poder. (DIAS, 2011, p. 62).

Diante de nossas experiências estéticas/sensíveis, o diálogo com as artes movimenta reflexões para além da complexidade formal e visual: nos estimula a perceber valores culturais, ideológicos e sociais que em outros contextos não perceberíamos, devido à sua natureza relacional e subjetiva. Neste contexto, nosso principal desafio corresponde às exigências da escrita acadêmica que nos impele à construção daquilo que chamamos de tecido provocativo, para dar conta das experiências vividas por meio da experiência estética, visual, sensorial. Isto é, nossas produções textuais no âmbito das artes derivam de processos de interpretação subjetiva. Partimos de relatos autobiográficos para encontrar nossos campos de pesquisa, esmiuçando nossos trânsitos entre um tema de interesse pessoal a configuração de uma tema de investigação acadêmica validado institucionalmente.

O marco teórico metodológico que respalda os projetos vinculados ao laboratório é atravessado por abordagens de cunho biográfico-narrativo, envolvendo perspectivas etnográficas, da bricolagem, A/R/Tográficas, Investigação Baseada nas Artes, Pedagogias Culturais, Processos de Mediação em distintos espaços expositivos (formais e não formais), Imaginário Social e Cultura Contemporânea, e ainda, abrindo-se ao caráter experimental e inventivo da pesquisa. Agrega projetos que visam processos colaborativos de aprendizagem e investigação no contexto da formação acadêmica, compartilhamento dos processos investigativos, ações embasadas por relações de reciprocidade e autonomia estudantil.

Como elementos para seguir a discussão, abordarei alguns projetos em andamento para dialogar neste texto: o primeiro, Referências Cruzadas: Exposições Itinerantes e Intercâmbio Cultural na América Latina ${ }^{2}$ vislumbra um importante processo de compartilhamento junto aos países latino-americanos, Argentina, Uruguai e México, como forma de resistência e fortalecimento destas parcerias interinstitucionais. Em sua primeira edição no Brasil (no segundo semestre de 2017), fomentou o intercâmbio de produções artísticas decorrentes das preocupações e atravessamentos entre artistas/ professores e professoras/pesquisadores e pesquisadoras que atuam no campo das artes visuais nos diferentes países - aqui reunidos em diálogo e compartilhamento.

Outro projeto que irei abordar Desenho Expandido: abordagens transdisciplinares das representações do corpo, sinaliza a potencia da mobilidade e interdisciplinaridade no contexto de formação universitária. Neste caso, ao integrar docentes e estudantes que atuam nos cursos de artes visuais, artes cênicas, dança e medicina ${ }^{3}$, o projeto provocou movimentos de crise inventiva e de experimentação ao colocar em diálogo a experiência poética com a rigidez dos estudos anatômicos advindos das ciências biológicas. Vinculado ao Grupo de Estudos e Pesquisas em Arte e Medicina, teve como objetivo principal fomentar processos criativos, investigativos e interpretativos das representações do(s) corpo(s) humano(s) por meio do desenho a partir de proposições inter/ multi/ transdisciplinares. No projeto, o grupo percorreu variadas experiências de percepção do corpo: do contato com o corpo morto às percepções do próprio corpo durante as proposições cênicas e da dança.

Por fim, o projeto que nos ajuda nesta reflexão parte de experiências educativas desenvolvidas junto à Sala de Exposições Cláudio Carriconde: o NECCA (Núcleo Educativo Cláudio Carriconde). Criado em 2014, com o intuito de dar suporte às ações educativas desenvolvidas a partir de exposições ocorridas na respectiva sala de exposições, o grupo formado por cerca de 5 estudantes tem sido responsável por varias ações que envolvem não apenas o acompanhamen-

\footnotetext{
2- Projeto de Extensão com registro no SIE/UFSM. Coordenação: Lutiere Dalla Valle.

3- GEPAM - Grupo de Estudos e Pesquisas em Arte e Medicina. Desde 2014 vem desenvolvendo experiências de produção artística a partir do diálogo com docentes e estudantes do curso de Medicina da UFSM. O grupo tem acesso às aulas de microscopia, com exercícios de observação celular no microscópio, e observação de cadáveres no Departamento de Morfologia, com fins de percepção anatômica do corpo humano.
} 
to do público junto ao espaço expositivo, mas tem proposto outros desdobramentos, como o Arte na Mala ${ }^{4}$ e o Rota das Artes no Campus ${ }^{5}$, que têm colocado alternativas de acesso à produção artística local. Todos estes imbuídos pelo objetivo do compartilhamento, pela socialização e comprometimento com o contexto social da cidade de Santa Maria.

\section{Referências Cruzadas: intercâmbio e compartilhamento}

O Projeto Referências Cruzadas surgiu por volta de 2014 a partir das articulações de produções artísticas desenvolvidas pelos professores Carlos Coppa e Mabel Larrechart, da Argentina e México, respectivamente. Em 2016, a convite do Diretor do Instituto Escuela Nacional de Bellas Artes de Montevidéu - Uruguai, Fernando Miranda, realizei a exposição individual "La Translucidez del Cuerpo " na Sala Miguel Ángel Pareja (localizada neste instituto). Além disso, tive o privilégio de ser contatado pelo Professor Carlos Coppa - da Universidad Nacional La Plata - Argentina, para a possibilidade de integrar as ações que o grupo vinha desenvolvendo e que, naquele momento, inseria o Uruguai no movimento coletivo. Em seguida, iniciamos nossas correspondências, que vieram a concretizar-se com a exposição coletiva na Argentina, em setembro de 2017, na Sala Islas Malvinas - Centro Cultural Islas Malvinas - La Plata, Argentina. Com o tema Frontera, a mostra coletiva reuniu quase quarenta artistas advindos dos quatro países. No mês seguinte, a mostra trasladou-se para o Brasil, complementando o I Encontro Internacional Artes Visuais e suas I/Mediações: Conexões Interdisciplinares, realizado nas dependências da Universidade Federal de Santa Maria, promovido pelo Laboratório Artes Visuais e I/Mediações. Na ocasião, os idealizadores do projeto, Carlos Coppa e Mabel Larrechart abriram o evento com palestras, oficinas e rodas de conversa.

Neste contexto, os conceitos de território, limite e fronteira, de interesse fundamental para as referências cruzadas, consistiram em configuração metafórica para abordar os processos de formação dos grupos que se articulam entorno do projeto compartilhado, produzindo obras individuas e coletivas para integrar a mostra. Como artistas latino-americanos que vivem em territórios delimitados geograficamente por fronteiras, definidas enquanto diferenças, mas igualmente passíveis de conexões que nos fazem reconhecer-nos como cidadãos, a experiência permitiu-nos resignificar a ideia de uma fronteira cultural comum. A partir de nossas práticas artísticas, buscamos abordar a ideia de território e fronteira em múltiplas dimensões, tanto sociais e individuais, a partir do criativo, do estético e experimental, para pensar igualmente em uma perspectiva regional sobre os acontecimentos atuais nos cenários políticos e culturais. A nível acadêmico, o projeto ofereceu-nos elementos práticos à participação de outras áreas do conhecimento, tendo em vista a crescente demanda por projetos que promovam a troca de ideias e projetos de colaboração e cooperação entre os países. O formato proposto mostras de caráter modular, itinerante, compostas por trabalhos gráficos em papel ou tela, de fácil transporte e montagem - adquire potência articuladora ao favorecer-nos espaços de intercâmbio, de experiências coletivas e processos formativos. Da mesma forma, contribuiu-nos para fortalecer a produção artística docente e de pesquisadores e pesquisadoras que

Figuras 1 e 2 - Fragmento e visão geral da Exposição no Centro de Convenções da Universidade Federal de Santa Maria. Outubro de 2017 (arquivo pessoal)
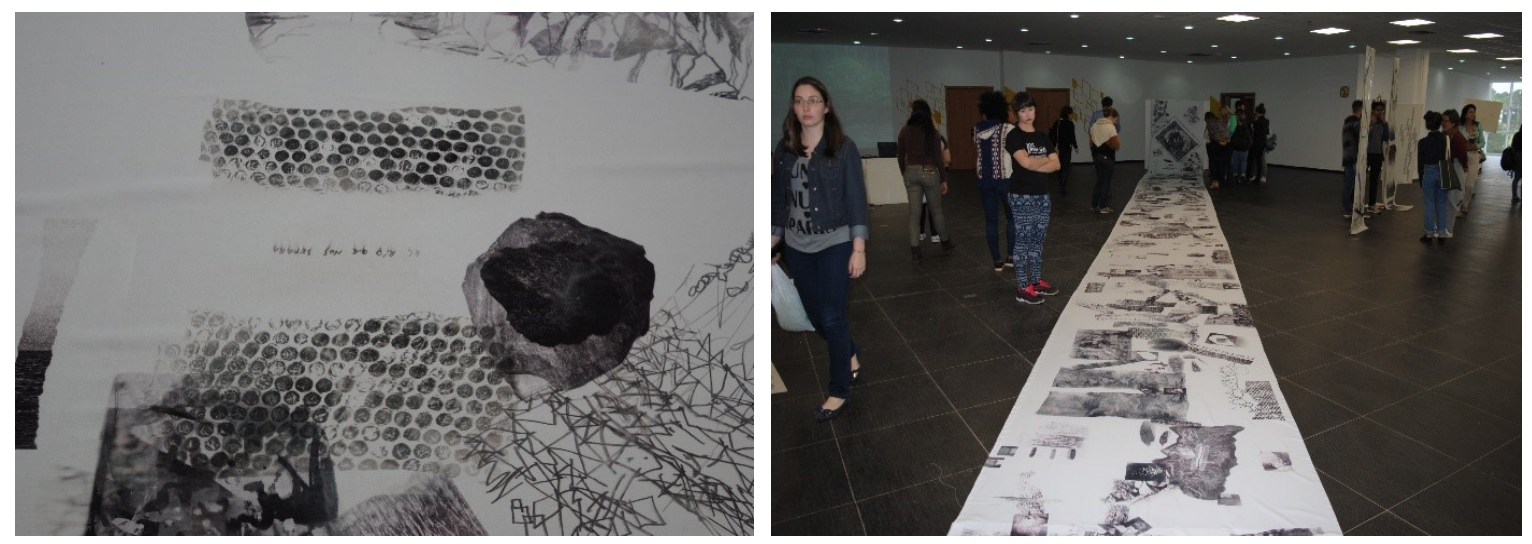

4- Art in Bag: Exposições Itinerantes é um projeto de extensão em que cerca de 30 obras dentro de uma grande mala - doações de estudantes do curso de artes Visuais da UFSM - percorre de forma itinerante instituições locais. As mostras são montadas em espaços disponibilizados pela instituição que as recebe, abrindo espaço para diálogos, oficinas e workshops.

5- Rota das Artes no Campus é um projeto de extensão que consiste no mapeamento das obras públicas distribuídas pelo Campus Central da UFSM e posteriormente, visitas guiadas com pequenos grupos, orientadas por acadêmicos do curso. A rota é definida previamente e conta com material informativo - mapa e informações básicas (autoria, técnica, período) das obras incluídas da rota de visitação. Ao finalizar a visitação, reflexão sobre lugar, espaço público e memória. 
atuam no Programa de Pós-Graduação em Artes Visuais PPGART/CAL/UFSM, estimulando a produção artística, a diluir fronteiras no âmbito das produções culturais da América Latina.

\section{Desenho Expandido: espaço de experimentação}

No âmbito da formação em artes visuais, temos investido no trânsito entre um referencial como ponto de partida ao processo contínuo de desconstrução, reconstrução e ressignificação dos elementos apreendidos pelo olhar e restituídos a partir de elaborações visuais. Estabelecemos um vínculo inicial com a materialidade para depois explorá-la e transformá-la de acordo com nossas intencionalidades poéticas/subjetivas: daquilo que produz em nós esta experiência de confrontação que mobiliza nosso pensar e o que reverbera.

Nossos encontros com os estudos anatômicos, em especial com os corpos inertes no Departamento de Morfologia da UFSM, têm nos permitido, entre outras coisas, refletir acerca de nossa própria existência ao observá-los e apreendê-los em nossas laborações poéticas. No contexto do Projeto de Estudos e Pesquisas em Arte e Medicina (GEPAM), desenvolvemos experiências estéticas e conhecimento científico/anatômico ao nos posicionamos como artistas pesquisadores a partir de abordagens multi e transdisciplinares. Desta forma, os estudos da anatomia humana sob o olhar atento e sensível da arte têm percorrido nossos processos investigativos com o objetivo de articular não apenas noções de representação gráfica daquilo que observamos, mas também a partir das potências artísticas que são impulsionadas a partir deste contato com a materialidade.

Figuras 3, 4 e 5 - Registros das sessões de observação, e experimentação e exercícios de percepção (arquivo pessoal)
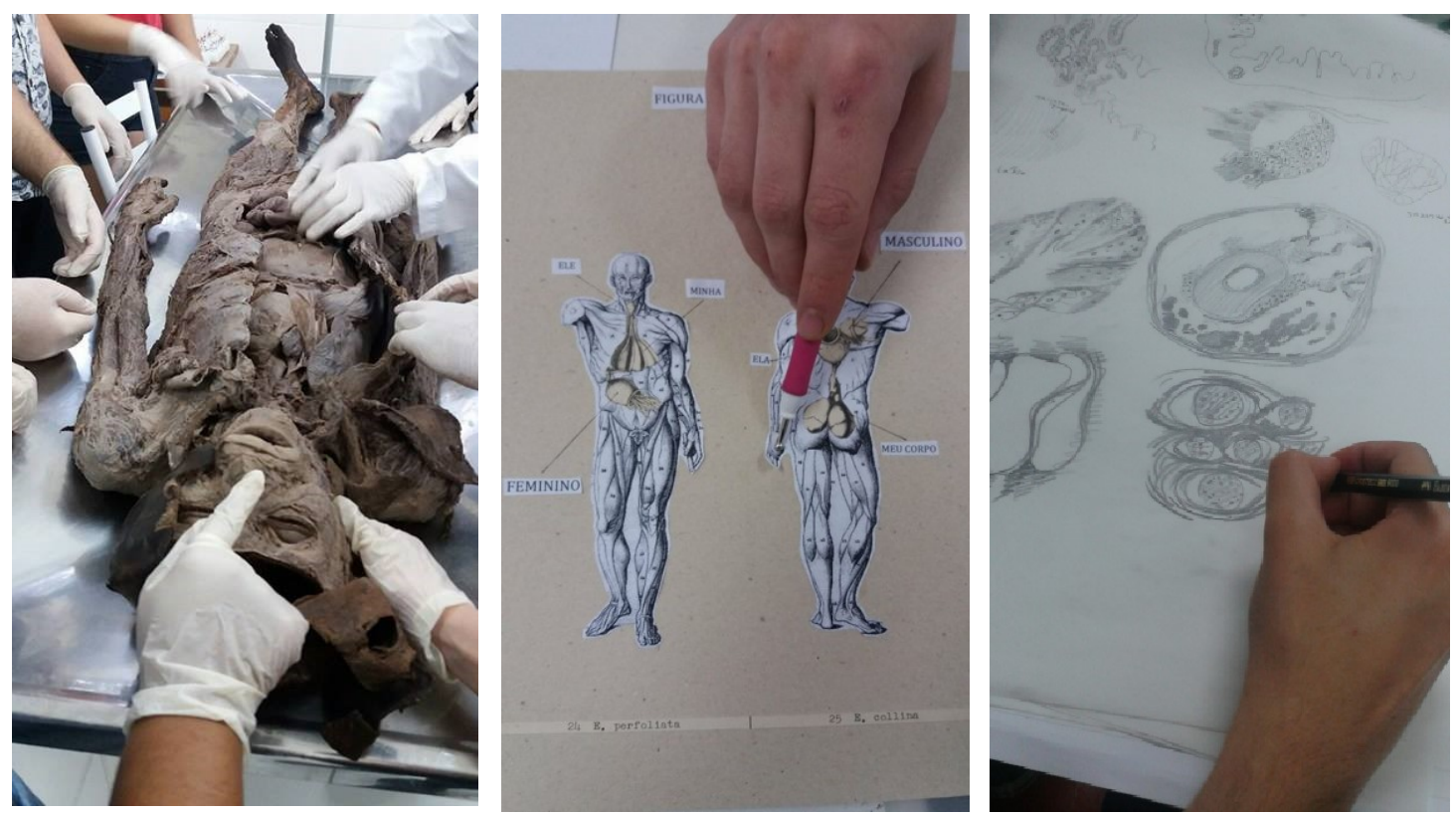

O desenho de anatomia artística surge, neste contexto, da necessidade de ancorarmos nossas inquietações diante da complexidade que constitui nossos corpos, bem como nossas próprias noções de ser e existir. Através da representação visual, temos recorrido a diversos recursos materiais e tecnológicos para materializar pensamentos acerca daquilo que captamos pelo aparato da visão e, que, a partir das concepções imaginarias e inventivas já apreendidas, devolvemos ao mundo simbolicamente. Enquanto síntese e cognição, o desenho surge a partir da confrontação de materialidades múltiplas, reflexos de pesquisa visual, de experimentação a partir daquilo que envolve percepção visual, tato, sincronia dos sentidos: tanto ao gesto espontâneo quanto ao que constrói posteriormente por meio da linha calculada, fortuita ou ainda pela destreza motriz que delimita espaços, formas, linhas, planos, estabelece fronteiras entre dentro e fora.

Sobre nossas capacidades inventivas, criar pode conectar-se ao ato de inventar, que nas palavras de Virginia Kastrup (2008) corresponde à ação de inventar problemas em torno das experiências de questionamento, pois, a aprendizagem inventiva promove rupturas que propõe deslocamentos cognitivos, isto é, nos incita a pensar em alternativas diversificadas.

Corpos fragmentados, entre visualidades macro e microscópicas: peles, ossos, carne, músculos, vísceras, tecidos, marcas, órgãos - como dialogamos com o corpo morto, fragmentado? Como pensamos nossos próprios corpos diante destas visualidades inertes? O que produz em nós artistas, docentes, investigadores? Que ressonâncias são produzidas a partir 
destes encontros? Que impactos são produzidos em nossas representações, noções de corpo, presença no mundo, finitude, perenidade? Quais são os desdobramentos desta experiência coletiva, compartilhada transdisciplinarmente? Como se dá o diálogo com o campo da representação diante dos códigos culturais que delimitam e delineiam corporeidades?

As questões permanecem ressoando sem respostas fixas, assim como no Livro de Anatomia Artística - contendo cerca de 70 desenhos realizados por estudantes e professores, poesias de uma acadêmica do curso de medicina e reflexões apontadas pelos docentes envolvidos - que pretende reunir as obras e propor uma versão poética dos conhecidos atlas de anatomia já legitimados pela área médica. Aqui, a proposta parte do campo da invenção, fazendo uso da ficção para criar órgãos e possibilidades de funcionalidade, estabelecendo relações entre imagem e palavra, entre arte, ciência, noções de verdade e invenção.

Atiçados pelas perguntas de Antônio Damásio (2012) (um dos autores referenciais do projeto), que dá segmento às provocações anteriores, será que nosso corpo sente o que nosso cérebro pensa? Será que nosso cérebro pensa o que o corpo sente? Questões que envolvem não apenas as formas como aprendemos a perceber noções de corpo e mente, igualmente nossas percepções cotidianas que nos atravessam em todas as instâncias da vida vivida: desde aquela que apresentamos diariamente por onde transitamos até aquela almejada, sonhada, que habita o campo dos sonhos, dos desejos, do imaginário. Lidar com os aspectos culturais que nos explicam os fenômenos entorno às noções de vida e morte, do corpo e sua presença cultural, tem provocado inúmeras conexões, perpassando desde à repulsa inicial às aproximações curiosas e propositivas que caracterizam o fazer artístico.

Desenho Expandido: abordagens transdisciplinares das representações do corpo consiste, portanto, em uma experiência que nos move a cada encontro rumo ao incerto, à espreita da potencia mobilizadora que reverbera a cada toque, a cada célula observada, onde um universo de possibilidades criativas se abre à nossa frente e nos permite exercitar nossa humanidade: sensível, atenta, inconformada e infinitamente inventiva.

\section{Zonas de contato: práticas de intervenção}

Aprender a partir da produção artística contemporânea com crianças e jovens tem sido um importante desafio na formação de acadêmicos e acadêmicas do curso de artes visuais. Em nossos grupos de pesquisa, ensino e extensão aos quais nos implicamos na Universidade em diálogo com a escola e outros espaços educativos, nos deparamos com um terreno movediço no qual nos posicionamos tendo em vista dois desafios principais: o primeiro deles no que tange a abordagem metodológica utilizada - que implica posições não hierárquicas, mas horizontais e permeadas pela curiosidade pueril; e em segundo, como convocar ao espaço de aprendizagem coletiva os conhecimentos prévios que cada sujeito carrega consigo e pode aportar em uma experiência coletiva.

De modo geral, as práticas abordadas vêm contribuindo com os processos de formação inicial dos/das estudantes ao possibilitar sua inserção nos ambientes educativos (formais e não formais). Neste contexto, tem sido relevante a adesão

Figuras 6, 7 e 8 - Visita guiada das obras públicas: Projeto Rota das Artes no Campus (arquivo pessoal).

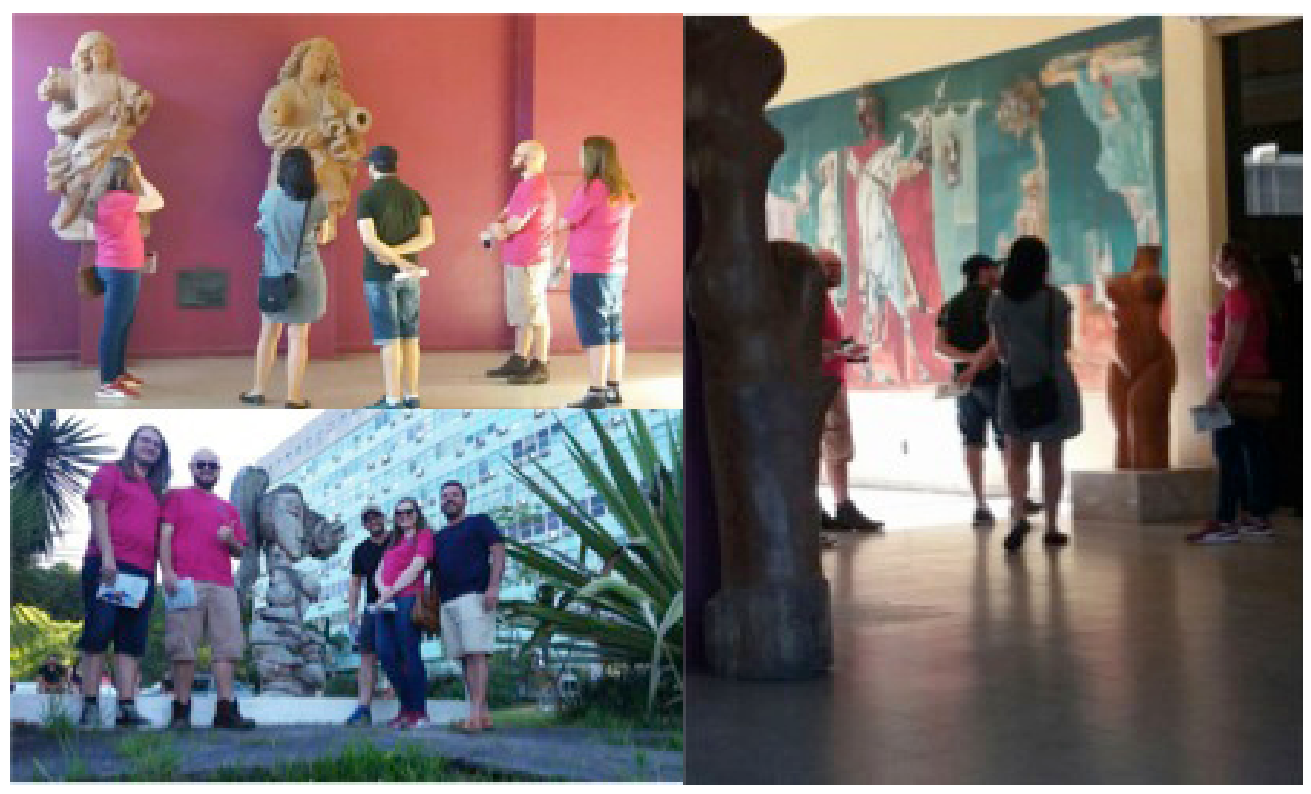


de estudantes do curso bacharelado aos projetos educativos vinculados às exposições artísticas - estudantes que não pretendem exercer a docência, porém almejam envolver-se com propostas educativas que vinculam a experiência artística e os espaços expositivos. Neste sentido, as ações desenvolvidas pelo LAVI/M tem sido de grande importância como lugar de experimentação, socialização e produção de conhecimento. Ações como o Arte na Mala e o Rota das Artes no Campus igualmente têm contribuído para que o vínculo com a comunidade local possa ser fortalecido, ampliando o número de acesso. As atuações do NECCA, que anteriormente ficavam restritas à Sala de Exposições no Centro de Artes e Letras da Universidade, passaram a percorrer instituições de ensino formal, receber diversos públicos para visitas guiadas às obras públicas (esculturas) instaladas pelo Campus Central da Universidade, favorecendo tanto experiências de interação, bem como aproximando a comunidade da produção artística local.

Ainda em fase inicial, as ações desenvolvidas pelos núcleos educativos de cada subprojeto antecipam pistas relevantes para pensar e propor processos e experiências coletivas de contato com as artes em distintos níveis - o que tem suscitado importantes trocas entre a comunidade estudantil e demais público envolvido. A partir dos encontros, o desafio configura-se desde o momento em que são definidas as obras, etapas e processos de cada ação, pois está implícito o caráter propositivo de quem propõe.

\section{Algumas considerações}

A organização e sistematização ao Laboratório de Artes Visuais e I/Mediações contribui com o fortalecimento da área das artes, sobretudo, no que tange o aprofundamento e a otimização dos recursos humanos e materiais para o desenvolvimento das experiências e investigações. Além disso, articula ações e práticas de formação colaborativa entre estudantes e professores, diálogos com outros grupos e laboratórios da instituição e igualmente de instituições externas.

Neste sentido, compreende mais que uma estrutura física adequada e disponível às reuniões de grupo, para o desenvolvimento de oficinas, workshops, atividades específicas, pois amplia-se à construção da identidade de um grupo que se reúne por afinidades, desenvolvendo projetos de cooperação com ênfase na busca acadêmica, compartilhando saberes do campo das artes, o que estimula a produção do conhecimento de forma ativa e expressiva.

Aborda-se a arte e sua potencialidade cognitiva a partir de experiências de aprendizagem onde as imagens são tomadas como artefatos visuais produzidos pelas culturas e difusoras de ideias que podem ser consentidas, projetadas, incorporadas e referendadas nas mais variadas situações da vida cotidiana. Para dar ênfase e sustentação a esta e outras discussões, a perspectiva dos estudos da cultura visual contribui para problematizarmos e refletirmos sobre o poder das imagens sobre nossas formas de compreensão e práticas sociais/culturais.

Pensando na potência das artes, podemos dizer que, enquanto articuladora, nos permitimos desenvolver práticas coletivas, híbridas, rompendo com a ideia tradicional da criação individual do/da artista pesquisador/pesquisadora. Enquanto mobilizadora, a arte desafia, incita o questionamento, desacomoda e instiga o pensamento, fomentou novos formatos educativos e investigativos ao revisitar paradigmas artísticos e culturais que operam durante a produção de sentidos. Por fim, sua dimensão educativa, abre espaço ao diálogo, à negociação, à formulação.

Como docente de uma instituição pública, percebo a potencialidade do campo artístico enquanto convite a revisitar crenças, verdades, sobretudo a problematizar a realidade construída, incitando-nos a refletir sobre nossas próprias ações cotidianas enquanto docentes. Implica-nos a não aceitar a realidade como imutável para pensar e colocar em movimento novas formas de transgressão. Além disso, assumir a docência como um processo em constante transformação, que se constrói pelas vias da complexidade humana, adaptando-se aos acontecimentos que atravessam a experiência.

\section{Referências}

DAMASIO, Antônio. O erro de Descartes: emoção, razão e o cérebro humano. São Paulo: Companhia das Letras, 2012.

DIAS, Belidson. 0 i/mundo da cultura visual. Brasília: Editora da Pós-Graduação em Arte da Universidade de Brasília, 2011.

HERNÁNDEZ, Fernando. Catadores da cultura visual: uma proposta para uma nova narrativa educacional. Porto Alegre: Mediação, 2007.

MIRZOEFF, Nicholas. Introducción a la cultura visual. Barcelona: Octaedro, 2003.

MITCHELL, W.; J .Thomas. Teoría de la imagen. Vol. 5. Ediciones Akal, 2009. 
RESENDE, José. (in); DERDYK, Edith (Org.). Disegno. Desenho. Desígnio. São Paulo: Editora Senac São Paulo, 2007. STOREY, John. Teoría cultural y cultura popular. Barcelona: Octaedro, 2002.

VAN MANEN, Max. Investigación educativa y experiencia vivida. Barcelona: Idea Books, 2003. 